\title{
Theoretical Study on the Oxidation of HCOOH on Pt Catalyst
}

\author{
Yingying Wang', Chengkuan Qin², and Lili Zhang ${ }^{1}$ \\ ${ }^{1}$ Shandong Vocational College of Light Industry, Zibo 255300, China \\ ${ }^{2}$ College of Aeronautical Engineering, Binzhou University, Binzhou, 256603, China \\ Corresponding author e-mail: 645774510@qq.com
}

Keywords: Pt Catalyst, Oxidation of $\mathrm{HCOOH}$, Theoretical

\begin{abstract}
New pathway for $\mathrm{HCOOH}$ decomposition to $\mathrm{CO}$ in the gas-phase on $\mathrm{Pt}(111)$ surface is proposed. By performing density functional theory calculations, this paper has evaluated the detailed reaction process, including energies and geometric structures of intermediates and transition states. The present theoretical calculation results show that the formation of $\mathrm{CO}$ on $\mathrm{Pt}(111)$ surface arises from $\mathrm{HCOOH}$ dehydration pathway. The present theoretical study rationalizes the early experimental findings well and provides insights into the deactivation problem of Pt catalyst in the process of $\mathrm{HCOOH}$ oxidation.
\end{abstract}

\section{Introduction}

As a promising alternative to $\mathrm{H}_{2}$ production, the electrocatalytic oxidation of formic acid $(\mathrm{HCOOH})$ on metal surfaces will play a very significant role in energy-efficient green power utilization in the future, especially in driving portable electronic appliances.[1] It is widely accepted that electrochemical oxidation of $\mathrm{HCOOH}$ proceeds via dual pathways, namely, dehydrogenation and dehydration.[2] The former pathway starts from the O-H bond dissociation converting to $\mathrm{CO}_{2}$. The latter pathway leads to $\mathrm{CO}$ via $\mathrm{C}-\mathrm{H}$ bond first scission. Generally, dehydrogenation or dehydration may predominate, depending on the properties of catalyst used. For HCOOH oxidation on Pt catalyst, the dehydrogenation pathway to yield $\mathrm{CO}_{2}$ is well-known to be facile.[3] However, Pt catalyst always loses a large amount of its activity during $\mathrm{HCOOH}$ oxidation owing to the poisoning adsorption of $\mathrm{CO}[4]$, indicating $\mathrm{HCOOH}$ dehydration with participation of $\mathrm{CO}$ intermediate should be considered as a potential pathway. Nevertheless, recent theoretical investigations for $\mathrm{HCOOH}$ decomposition on $\mathrm{Pt}(111)$ unanimously reported that the dehydration pathway involves a much higher barrier than the dehydrogenation one (1.39-1.80 eV vs. 0.45-0.79 $\mathrm{eV}$ ) [5]. Thus $\mathrm{HCOOH}$ should be directly oxidized to final product $\mathrm{CO}_{2}$ rather than to the adsorbed CO intermediate. This is clearly inconsistent with the observed facile CO poisoning of $\mathrm{Pt}(111)$. Now, the question is where $\mathrm{CO}$ originates mainly from in the process of the $\mathrm{HCOOH}$ oxidation on Pt catalyst. Answering this issue would provide insights into the deactivation problem. By performing density functional theory (DFT) calculations, we show the potential energy surface details of $\mathrm{CO}$ formation according to the possible mechanism which involving subtly different reaction intermediates and transition states are explicitly obtained. We hope to understand the easy poisoning effects on Pt catalyst.

\section{Computational Details}

DFT periodic slab calculations were performed using the CASTEP package[6] with ultrasoft pseudopotentials and the spin polarized generalized gradient approximation (GGA) exchangecorrelation functional proposed by Perdew, Burke, and Ernzerhof (PBE)[7]. The Kohn-Sham oneelectron states are expanded in a plane wave basis set up to a cutoff energy of $400 \mathrm{eV}$. MonkhorstPack meshes with $2 \times 2 \times 1 \mathrm{k}$-grid sampling in the surface Brillouin zone are used. The criteria for energy and maximum force convergence used are $2.0 \times 10^{-5} \mathrm{eV} /$ atom and $0.05 \mathrm{eV} / \AA$. With this setting we get the lattice constant of bulk Pt as $3.96 \AA$, which agrees well with the experimental 
finding, $3.92 \AA$. .[8] $\mathrm{HCOOH}$ has the trans and the cis forms, depending on the orientation of the hydroxyl group (-OH). Experiment has confirmed that the trans conformer is the dominant in the gas phase, and the energy difference between the two conformers is about $0.17 \mathrm{eV}$.[9] In this study, the energy of the trans form was calculated to be $0.15 \mathrm{eV}$ lower than that of the cis form, which is in good agreement with the experimental value.

Previous work comparing 3 and 5 layer metal slabs found little change on the structural water.[10] Therefore, $\mathrm{Pt}(111)$ surface is modeled by a periodic slab containing three atomic layers with full relaxation of the uppermost layer. A $\mathrm{p}(3 \times 3)$ unit cell with 9 metal atoms per layer is used in this study. The vacuum separation between periodically repeated slabs is $10 \AA$, which is large enough to avoid interactions between slabs. The transition states (TSs) are searched for with the linear and quadratic synchronous transit (LST/QST) complete search.[11]

The adsorption energies are defined as $E_{\text {ad }}=E_{\text {adsorbate }}+E_{\text {surf }}-E_{\text {total }}$, where $E_{\text {adsorbate }}, E_{\text {surf }}$, and $E_{\text {totel }}$ refer to the energies of free adsorbate, the clean surface, and the adsorbed system, respectively. For a CO formation reaction, the activation barrier $\left(E_{a}\right)$ is calculated by $E_{a}=E_{T S}-E_{\text {totel }}$, respectively, where $\mathrm{E}_{\mathrm{TS}}$ and $\mathrm{E}_{\text {totel }}$ are the transition state and adsorbed system, respectively.

\section{Result and Discussion}

Intially, it should be emphasized that most settings and parameters described in the model and method section, including the $\mathrm{p}(3 \times 3)$ supercell, three-layer slab, $2 \times 2 \times 1$ k-point, $10 \AA$ vacuum zone, and the energy cutoff of $400 \mathrm{eV}$, have been extensively confirmed in literature to be accurate enough for the periodic slab calculations on noble metal surfaces[12]. To further calibrate the selected method, we here compare several calculated results with available experimental values. (i) The $\mathrm{O}-\mathrm{H}, \mathrm{C}-\mathrm{H}, \mathrm{C}=\mathrm{O}$, and $\mathrm{C}-\mathrm{O}$ bond distances in a free $\mathrm{HCOOH}$ are calculated to be 0.986, 1.105, 1.218, and $1.354 \AA$, while the corresponding experimental values[13] are 0.972, 1.097, 1.202, and $1.343 \AA$, respectively. (ii) The calculated largest adsorption energy of $\mathrm{HCOOH}$ on $\mathrm{Pt}(111)$ surface is $0.41 \mathrm{eV}$, while previous theoretical result is $0.43 \mathrm{eV}[14]$. These data show good agreements with the calculated results and available reference values, indicating sufficiency of the model and method used. In addition, in the present work our main concern is the accuracy of the relative energies for several adsorption and decomposition processes considered. Energy errors for the initial state and the final state of a chemical process are almost the same and can counteract each other. It should be mentioned that GGA DFT is not accurate enough to describe long-range attractive contribution to van der Waals interactions although it can handle the dispersion force near the van der Waals minimum.[15] However, it is expected that systematic errors resulting from long-range van der Waals interactions are mostly counteracted in our relative energy calculations. Therefore the deficiency of GGA DFT in describing van der Waals interactions may not change the present conclusions, which are drawn out from calculated relative energy differences of intermediates and transition states involved during the $\mathrm{HCOOH}$ decomposition. Thus, we consider that the conclusion to be drawn in the present work based on calculated relative energies is reliable.
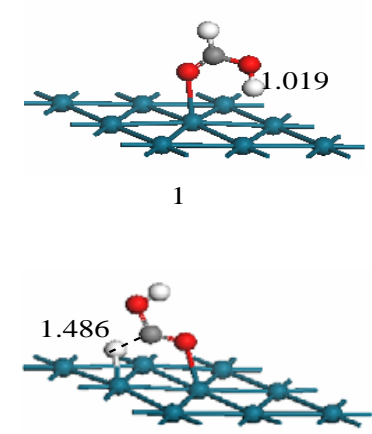

$\mathrm{TS}_{2-3}$

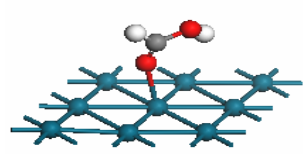

$\mathrm{TS}_{1-2}$

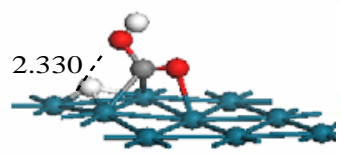

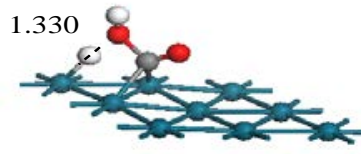

$\mathrm{TS}_{3-4}$
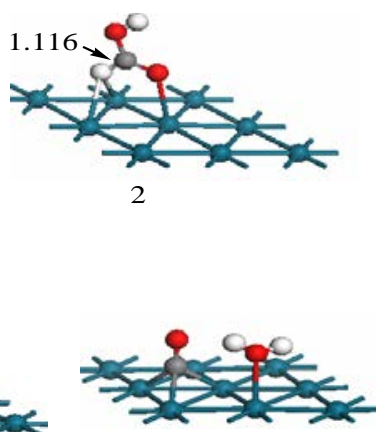

4

Figure 1. Optimized geometries for the intermediates and the transition states involved during CO formation along the path for $\mathrm{HCOOH}$ oxidation. For clarity, only one layer is illustrated. Distances are in angstroms. 
We then perform calculations of the possible decomposition route, and locate various intermediates and TSs along the pathway. Their structures with the key geometrical parameters are shown in Figure 1. The relevant potential energy surface (PES) profile along the reaction coordinate in Figure 2, where the sum of the energy of the isolated reactants ( $\mathrm{HCOOH}$ and the clean Pt surface) is taken as zero energy.

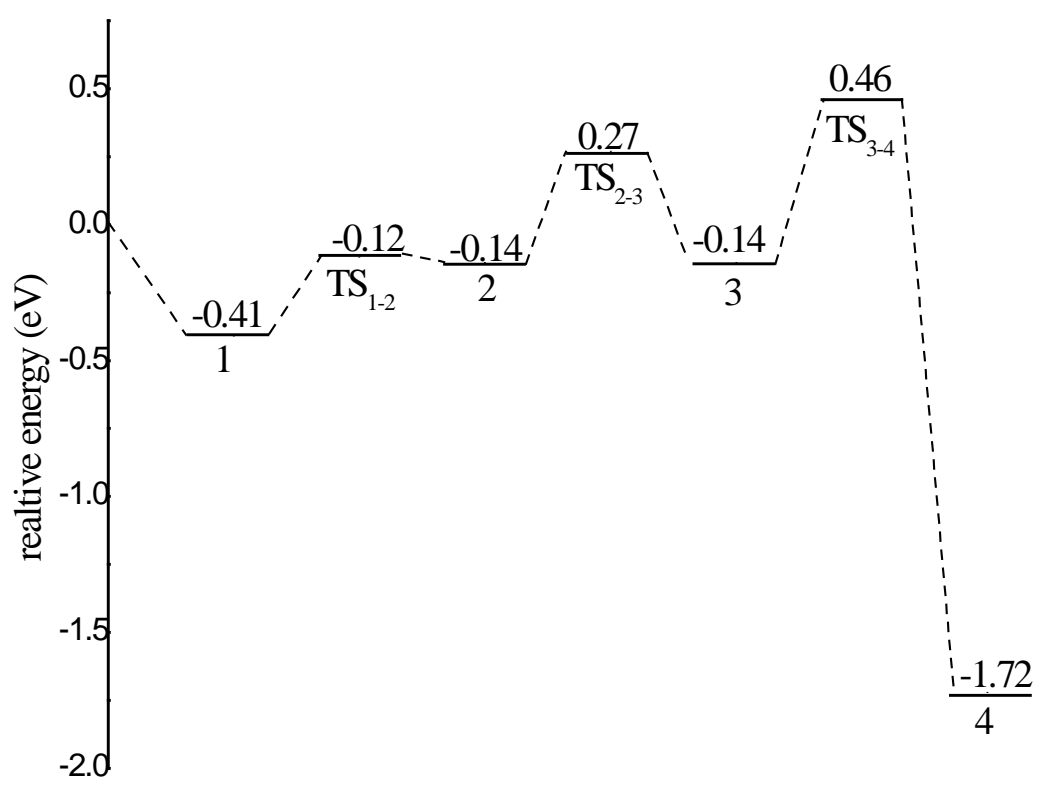

Figure 2. Calculated potential energy surface profile for forming $\mathrm{CO}$ along the reaction paths

From our calculations, the path involves two elementary steps: the breaking of C-H bond and the formation of $\mathrm{CO}$ and $\mathrm{H}_{2} \mathrm{O}$ molecules. We first obtained the possible molecular adsorbed form of formic acid (labled structure 1 in Figure 1), which is the starting point for the subsequent mechanism. In structure 1, the carbonyl oxygen is bonded on top of one Pt atom and the $\mathrm{OH}$ points asymmetrically to two neighboring Pt atoms. This structure is analogous to the bidentate structures, which were observed for formate on the Pt(111) surface.[16] Our result is consistent with other theoretical studies reported in literature.[14] The most evident change in structure 1 is the O-H bond length $(1.019 \AA)$, which is longer than that of an isolated $\mathrm{HCOOH}(0.986 \AA)$. The computed adsorption energy for $1 \mathrm{a}$ is $0.41 \mathrm{eV}$, which is agreement with the result obtained in the other theoretical studies.[17] The $\mathrm{C}-\mathrm{H}$ bond is difficult to be activated in structure 1 since it points away from the Pt surface. In order to dissociate this bond, $\mathrm{HCOOH}$ must be clockwise rotated about the axis of the $\mathrm{C}=\mathrm{O}$ bond, making the carbonyl hydrogen atom point to the surface (structure 2), which requires $0.29 \mathrm{eV}$. Configuration 2 shows an adsorption geometry in which the carbonyl oxygen interacts strongly and the carbon $\mathrm{H}$ weakly with the surface with $\mathrm{E}_{\mathrm{ad}}=0.14 \mathrm{eV}$. The C-H bond length is slightly increased to $1.116 \AA$ from a gas phase value of $1.105 \AA$, while no similar increase is observed in structure 1 . If the $\mathrm{C}-\mathrm{H}$ bond of formic acid in structure 2 is activated, $\mathrm{COOH}$ forms in structure 3. Structures 2 and 3 are connected by transition state $\mathrm{TS}_{2-3}$, where the $\mathrm{C}-\mathrm{H}$ bond stretches to $1.486 \AA$. The reaction barrier of the $\mathrm{C}-\mathrm{H}$ bond breaking is $0.41 \mathrm{eV}$. After the $\mathrm{O}-\mathrm{H}$ bond scission, breaking the $\mathrm{C}-\mathrm{OH}$ bond via the transition state $\mathrm{TS}_{3-4}$ can generate the product 4 , $\mathrm{CO}$ and $\mathrm{H}_{2} \mathrm{O}$. The distance between $\mathrm{H}$ atom and $\mathrm{O}$ atom of $\mathrm{OH}$ group are shorted to $1.330 \AA$ in $\mathrm{TS}_{3-4}$ from $2.330 \AA$ in structure 3 . The activation barrier for this elementary reaction is $0.60 \mathrm{eV}$. From Figure 2, we can see that this step is the rate-determining step in this path. This barrier is lower than the $0.88 \mathrm{eV}$ required to dissociate the $\mathrm{C}-\mathrm{OH}$ bond when $\mathrm{HCOOH}$ is absorbed as an unstable cis-HCOOH species.[17] Not surprisingly, the difference between these two barriers is due to the different adsorption structures of $\mathrm{HCOOH}$.

Comparing with the previous studies,[14] it should be noted that $\mathrm{O}-\mathrm{H}$ or $\mathrm{C}-\mathrm{H}$ bond breaking is 
dependent on adsorbed $\mathrm{HCOOH}$ orientation, meaning each orientation will have different possible intermediates and pathways. From the potential energy surface of pathway shown in Figure 2, the rate-determining step in this path is $0.60 \mathrm{eV}$, which is lower than those reported in the literature,[14] indicating that $\mathrm{CO}$ formation in the gas phase from the decomposition of formic acid on $\mathrm{Pt}(111)$ is energetically favorable, which agrees well with experimental findings.[4]

\section{Conclusion}

The formation of $\mathrm{CO}$ on $\mathrm{Pt}(111)$ surface during $\mathrm{HCOOH}$ decomposition under gas-phase conditions has been studied in detail with DFT. Our aim is to hunt a new possible pathway that lead to the formation of CO to rationalize the poisoning of Pt-based catalysts. The proposed pathway for $\mathrm{CO}$ formation involves the rate-determining step barrier of $0.60 \mathrm{eV}$. The present result imply that the reaction of $\mathrm{HCOOH}$ decomposition to $\mathrm{CO}$ becomes favorable thermodynamically, which rationalize the experimental fact well.

\section{Acknowledgments}

This work was financially supported by Science and Technology Development Plan of Zibo (No. 2016kj010034), Scientific Research Foundation of Binzhou University (Grant BZXYL1403) and a Project of Shandong Province Higher Educational Science and Technology Program (J15LC56).

\section{References}

[1] S. Uhm, H.J. Lee, Y. Kwon, J. Lee, A Stable and Cost-Effective Anode Catalyst Structure for Formic Acid Fusel Cells, Angew. Chem. Int. Ed. 47 (2008) 10163-10166.

[2] A.C. Chen, P. Holt-Hindle, Platinum-Based Nanostructured Materials: Synthesis, Properties, and Applications. Chem. Rev. 110 (2010) 3767-3804.

[3] W. Gao, J.A. Keith, J. Anton, T. Jacob, Theoretical Elucidation of the Competitive Electrooxidation Mechanisms of Formic Acid on Pt(111), J. Am. Chem. Soc. 132 (2010) 18377-18385.

[4] T. Iwasita, X.H. Xia, E. Herrero, H.D. Liess, Early Stages during the Oxidation of HCOOH on Single-Crystal Pt Electrodes As Characterized by Infrared Spectroscopy, Langmuir 12 (1996) 42604265.

[5] H.F. Wang, Z.P. Liu, Formic Acid Oxidation at Pt/H2O Interface from Periodic DFT Calculations Integrated with a Continuum Solvation Model, J. Phys. Chem. C 113 (2009) 17502.

[6] M.C. Payne, M.P. Teter, D.C. Allen, T.A. Arias, J.D. Joannopolous, Iterative Minimization Techniques for Ab Initio Total-Energy Calculations: Molecular Dynamics and Conjugate Gradients. Rev. Mod. Phys. 64 (1992) 1045-1097.

[7] J.P. Perdew, K. Burke, M. Ernzerhof, Generalized Gradient Approximation Made Simple. Phys. Rev. Lett. 77 (1996) 3865-3868.

[8] D.R. Lide, CRC Handbook of Chemistry and Physics, 83rd ed., CRC Press, New York, 2002.

[9] W.H. Hocking, The other Rotamer of Formic Acid, cis-HCOOH. Z. Naturforsch. 31a (1976) $1113-1121$.

[10] C.D. Taylor, R.G. Kelly, M. Neurock, First-Principles Calculations of the Electrochemical Reactions of Water at an Immersed $\mathrm{Ni}(111) / \mathrm{H}_{2} \mathrm{O}$ Interface. J. Electrochem. Soc. 153 (2006) E207-E214.

[11] N. Govind, M. Petersen, G. Fitzgerald, S.D. King, J. Andzelm, A Generalized Synchronous Transit Method for Transition State Location. Comput. Mater. Sci. 28 (2003) 250-258.

[12] G. Wang, A.K. John, A. Josef, J. Timo, Oxidation of Formic Acid on the Pt(111) Surface in the 
Gas Phase. Dalton Trans. 39 (2010) 8450-8456.

[13] A. Staykov, T. Kamachi, T. Ishihara, K. Yoshizawa, Theoretical Study of the Direct Synthesis of H (2) O (2) on Pd and Pd/Au Surfaces. J. Phys. Chem. C 112 (2008) 19501-19505.

[14] Y.Y. Qi, R.X. Zhu, D.J. Zhang, Adsorption behaviors of monomer and dimer of formic acid on Pt (111) in the absence and presence of water J. Mol. Model. 20 (2014) 2264

[15] F. Tran, J. Weber, T.A. Wesolowski, F. Cheikh, Y. Ellinger, F. Pauzat, Physisorption of Molecular Hydrogen on Polycyclic Aromatic Hydrocarbons: A Theoretical Study. J. Phys. Chem. B 106 (2002) 8689-8696.

[16] J. Kitchin, J.K. Nørskov, M. Barteau, J. Chen, Modification of the surface electronic and chemical properties of Pt(111) by subsurface 3d transition metals J. Chem. Phys. 120 (2008) 10240

[17] G. Wang, A.K. John, A. Josef, J. Timo, Oxidation of Formic Acid on the Pt(111) Surface in the Gas Phase. Dalton Trans. 39 (2010) 8450-8456. 